\title{
Economics and the Endangered Species Act: Critical Habitat Designation for Pacific Northwest Salmon and Steelhead
}

\author{
MARK L. PLUMMER
}

Under the Endangered Species Act (ESA), NOAA's National Marine Fisheries Service (NMFS) is responsible for protecting and recovering species of marine fish, mammals, and plants that it determines to be in danger of extinction. As one of its responsibilities, the agency designates critical habitat for these species. Critical habitat consists of areas determined to have biological or physical features important to the conservation of the species. In areas designated as critical habitat, federal agencies must ensure that their actions do not adversely modify the habitat.

Mark Plummer (1954-2014) was with the Conservation Biology Division, Northwest Fisheries Science Center, National Marine Fisheries Service, NOAA, 2725 Montlake Blvd. East, Seattle, WA 98112-2097. In 2015 the NWFSC honored his career with a memorial symposium (https:// www.nwfsc.noaa.gov/news/events/symposia/ mark_plummer/). The views expressed in this paper were those of the author and do not necessarily represent the position of the National Marine Fisheries Service, NOAA.

doi: dx.doi.org/10.7755/MFR.77.3.9
The process for designating critical habitat is unusual in that it actually requires the consideration of economic impacts. The ESA gives NMFS the option to exclude individual areas from designation if the costs of designation outweigh the benefits of designation. The costs stem from the economic costs of changing federal activities to comply with the ESA in this regard, while the benefits come from the additional conservation achieved in an area that has been designated.

For west coast Pacific salmon, Oncorhynchus spp., and steelhead trout, Oncorhynchus mykiss, listed under the ESA, NMFS devised a framework to balance the economic costs and conservation benefits of critical habitat designation. NMFS used watershed units as mapped by the U.S. Geological Survey to define the individual areas for consideration.

For each watershed, an estimate was made of the economic costs of designation, which came from considering how the designation would affect ten types of federal activities that poten- tially occurred in a watershed. Teams of biologists then determined the conservation benefit of designation, expressed as a relative score (high, medium, low) for the potential value of protecting the watershed to conserve the species.

Finally, a threshold cost level was chosen that governed the process for excluding a watershed, with higher thresholds chosen for higher levels of conservation benefit. If the estimated costs in a watershed were greater than the appropriate threshold, the watershed was then eligible for exclusion. This process enabled the agency to identify and exclude potential critical habitat areas that entailed high economic costs and low conservation benefits, making the designation of critical habitat more cost-effective (Plummer, 2007). Following this approach, NMFS excluded 106 watersheds from critical habitat designation.

Plummer, M. 2007. Welcome to the data-poor real world: incorporating benefit-cost principles into environmental policymaking. Res. Law Econ. 23:103-130. 